\title{
On $p$-harmonic measures in half-spaces
}

\author{
José G. Llorente ${ }^{1}(\mathbb{D})$ Juan J. Manfredi ${ }^{2} \cdot$ William C. Troy ${ }^{2} \cdot J^{\prime}$ ang-Mei Wu ${ }^{3}$
}

Received: 14 September 2018 / Accepted: 28 December 2018 / Published online: 19 January 2019 (c) Fondazione Annali di Matematica Pura ed Applicata and Springer-Verlag GmbH Germany, part of Springer Nature 2019

\begin{abstract}
For all $1<p<\infty$ and $N \geq 2$ we prove by using ODE shooting techniques that there is a constant $\alpha(p, N)>0$ such that the $p$-harmonic measure in $\mathbb{R}_{+}^{N}$ of a ball of radius $0<\delta \leq 1$ in $\mathbb{R}^{N-1}$ is bounded above and below by a constant times $\delta^{\alpha(p . N)}$. We provide explicit estimates for the exponent $\alpha(p, N)$.
\end{abstract}

Keywords $p$-Laplacian $\cdot p$-Harmonic measure $\cdot$ Shooting method

Mathematics Subject Classification 34B40 $\cdot 34 \mathrm{C} 11 \cdot 35 \mathrm{~J} 60$

\section{Introduction}

In this paper we study $p$-harmonic measures, which in the case $p=2$, are harmonic functions defined as follows: given a domain $\Omega \subset \mathbb{R}^{N}$, a point $x \in \Omega$ and a subset $E \subset \partial \Omega$, the harmonic measure of $E$ from $x$ in $\Omega$, denoted $\omega(E, x, \Omega)$, is the value at $x$ of the harmonic function $\omega(E, x, \Omega)$ satisfying

The first author was supported by Grants MTM2017-85666-P, 2017 SGR 395 (Spain). The fourth author was supported by Simon Foundation Grant \#353435.

$凶 \quad$ José G. Llorente

jgllorente@mat.uab.cat

Juan J. Manfredi

manfredi@pitt.edu

William C. Troy

troy@math.pitt.edu

Jang-Mei Wu

jmwu@illinois.edu

1 Departament de Matemàtiques, Universitat Autònoma de Barcelona, 08193 Bellaterra, Barcelona, Spain

2 Department of Mathematics, University of Pittsburgh, 301 Thackeray Hall, Pittsburgh, PA 15260, USA

3 Department of Mathematics, University of Illinois at Urbana-Champaign, 1409 West Green Street, Urbana, IL 61801, USA 


$$
\omega(E, x, \Omega)=\left\{\begin{array}{l}
1 \text { for } x \in E \\
0 \text { for } x \in \partial \Omega \backslash E
\end{array}\right.
$$

when $\Omega$ and $E$ are sufficiently regular. It follows from the linearity of the Laplace operator that for fixed $x, \omega(., x, \Omega)$ is a probability measure on $\partial \Omega$ and from the Harnack property $\omega(., x, \Omega)$ and $\omega(., y, \Omega)$ are mutually absolutely continuous. The study of the metric properties of harmonic measure and its connection to Hausdorff measures on $\partial \Omega$ has played a fundamental role in the development of modern geometric function theory and it is related to several branches of mathematics such as PDEs, probability, potential theory and dynamical systems among others.

Our main focus is on the case $p \neq 2$ when the relevant differential equation is the nonlinear $p$-Laplace equation

$$
\operatorname{div}\left(|\nabla u|^{p-2} \nabla u\right)=0 \quad(1<p<\infty) .
$$

Weak solutions of (1.1) in the Sobolev space $W_{\text {loc }}^{1, p}(\Omega)$ are called $p$-harmonic functions in $\Omega$. When $p \rightarrow \infty$ we formally obtain another differential operator which is not in divergence form, the so-called infinity Laplacian $\triangle_{\infty}$ given by

$$
\triangle_{\infty} u=\sum_{i, j=1}^{N} u_{x_{i}} u_{x_{j}} u_{x_{i} x_{j}},
$$

and solutions of $\triangle_{\infty} u=0$ in the viscosity sense are called infinity harmonic functions.

For $p \neq 2$ the definition of $p$-harmonic measure $\omega_{p}(., x, \Omega)$ follows the above potential theoretic approach (see [9]). Because of the nonlinearity $p$-harmonic measures are more difficult to handle and lack some of the nice properties available in the linear case $p=2$. It is important to recognize that $\omega_{p}($.$) is no longer a measure, not even at the zero level (see [14]).$ In the case $p=2$ it is easy to estimate the harmonic measure of subsets of the boundary of the ball or the upper half-space, due to the explicit expression of the Poisson kernel in a ball or a half-space. The situation is more complicated when $p \neq 2$, even for simple subsets of the boundary like spherical caps or half-space balls. One of the first results in this direction was obtained by Peres et al. [18]. They proved that

$$
\omega_{\infty}\left(C_{\delta}, 0, \mathbb{B}_{N}\right) \approx \delta^{1 / 3}
$$

where $\mathbb{B}_{N}$ denotes the unit ball in $\mathbb{R}^{N}, C_{\delta}$ is any spherical cap of radius $\delta$ and $\omega_{\infty}$ stands for the $\infty$-harmonic measure. The proof of (1.3) is based on two facts. First, because of rotational invariance, the problem can be reduced to two dimensions. The second is the use of quasiradial singular $\infty$-harmonic functions obtained by Aronsson [2], which are of the form $r^{k} f(\phi)$ where $(r, \phi)$ denote polar coordinates in the plane and $k=-1 / 3$. The function $r^{-1 / 3} f(\phi)$ plays the role of the Poisson kernel in the case $p=\infty$ and is used to estimate $\omega_{\infty}\left(C_{\delta}, 0, \mathbb{B}_{N}\right)$.

Next, we recall properties of the Poisson kernel $P\left(x, z^{\prime}\right)$ in the upper half-space $\mathbb{R}_{+}^{N}$ defined by

$$
P\left(x, z^{\prime}\right)=\frac{x_{N}}{\left(\left|x^{\prime}-z^{\prime}\right|^{2}+x_{N}^{2}\right)^{N / 2}}
$$

Here $z^{\prime} \in \mathbb{R}^{N-1}$ and $x=\left(x^{\prime}, x_{N}\right) \in \mathbb{R}_{+}^{N}$, where $x^{\prime} \in \mathbb{R}^{N-1}$, and $x_{N}>0$. It is well known that $P\left(., z^{\prime}\right)$ is positive and harmonic in $\mathbb{R}_{+}^{N}$ and has unrestricted boundary values 0 on $\mathbb{R}^{N-1} \backslash\left\{z^{\prime}\right\}$ and nontangential limits $+\infty$ when $x \rightarrow z^{\prime}$, for every $z^{\prime} \in \mathbb{R}^{N-1}$. Set $z^{\prime}=0$ 
and define $P(x)=P(x, 0)$. Since $P$ depends only on the distance to the origin $r=|x|$ and the azimuth angle $\theta$ formed by $x$ and the positive $x_{N}$-axis, we write

$$
P(x)=P(r, \theta)=r^{-(N-1)} \cos \theta,
$$

so the singularity at the origin is of order $r^{-(N-1)}$. In the case $p \neq 2$ the analogue of the Poisson kernel is played by quasiradial functions $u=r^{k} f(\theta)$ with $k<0$ and the following assumptions on $f$ :

$$
\begin{aligned}
& f:[0, \pi / 2] \rightarrow \mathbb{R} \text { is positive and decreasing, } \\
& f \in C^{2}[0, \pi / 2], \\
& f(0)=1, f^{\prime}(0)=0, f(\pi / 2)=0 \text { and }-\infty<f^{\prime}(\pi / 2)<0 .
\end{aligned}
$$

\subsection{Previous results}

Using Aronsson's positive singular quasiradial $p$-harmonic functions in the plane, Lundström and Vasilis [13] obtained sharp estimates for $p$-harmonic measures in planar domains satisfying appropriate regularity assumptions. They proved in particular that

$$
\begin{aligned}
& \omega_{p}\left(I_{\delta}, i, \mathbb{R}_{+}^{2}\right) \approx \delta^{q} \quad \text { if } 1<p<\infty \quad \text { and } \\
& \omega_{p}\left(A_{\delta}, 0, \mathbb{D}\right) \approx \delta^{q} \quad \text { if } p \geq 2,
\end{aligned}
$$

where

$$
q=\frac{3-p+2 \sqrt{p^{2}-3 p+3}}{3(p-1)},
$$

$I_{\delta}$ is the interval $[-\delta, \delta] \subset \mathbb{R}, A_{\delta}$ is an arc of length $\delta$ on the unit circle and $\mathbb{D}$ is the unit disk.

The case $p=N$ is special because $N$-harmonic functions in $\mathbb{R}^{N}$ are conformally invariant. Hirata [10] proved the estimate $\omega_{N}\left(B(\xi, \delta) \cap \partial \Omega, x_{0}, \Omega\right) \approx \delta$ if $\Omega$ is a $C^{1,1}$ domain in $\mathbb{R}^{N}$. Recently, DeBlassie and Smits [5,6] have made further contributions in the case $N \geq 3$ and general $p$. The key point in [5] is the computation of $\triangle_{p} u$ where $u=r^{k} f(\theta)$ is a quasiradial function. Up to a positive factor $\triangle_{p} u$ is given by the following differential expression

$$
\begin{array}{r}
{\left[(p-1)\left(f^{\prime}\right)^{2}+k^{2} f^{2}\right] f^{\prime \prime}+k[(2 p-3) k+N-p] f\left(f^{\prime}\right)^{2}+} \\
k^{3}[k(p-1)+N-p] f^{3}+(N-2)\left[\left(f^{\prime}\right)^{2}+k^{2} f^{2}\right] f^{\prime} \cot \theta .
\end{array}
$$

Observe that if $f$ is decreasing and $\theta \in[0, \pi / 2]$, the fourth term in (1.11) is negative. Thus, solutions to the reduced equation

$$
\begin{aligned}
& {\left[(p-1)\left(f^{\prime}\right)^{2}+k^{2} f^{2}\right] f^{\prime \prime}+k[(2 p-3) k+N-p] f\left(f^{\prime}\right)^{2}+} \\
& k^{3}[k(p-1)+N-p] f^{3}=0
\end{aligned}
$$

provide $p$-superharmonic functions. This approach gives upper bounds for $p$-harmonic measure in the ball or the upper half-space [5] (see Sect. 2). In [6] another reduction of (1.11) is used to obtain additional upper and lower bounds under appropriate restrictions on $N, k$ and $p$, which are complementary to those of [5]. 


\subsection{Main results}

Our first main result provides explicit estimates for the $p$-harmonic measure of a ball on the boundary of a half-space. Because of the translation invariance of the $p$-harmonic equation and the Harnack property we assume that the ball is centered at the origin and the base point lies at distance one above the center.

Theorem 1.1 Let $N \geq 2,1<p<\infty, 0<\delta \leq 1, B_{\delta} \subset \mathbb{R}^{N-1}$ the ball in $\mathbb{R}^{N-1}$ centered at the origin of radius $\delta$ and $x_{0}=(0, \ldots, 0,1)$.

(1) If $\mathbf{1}<\boldsymbol{p} \leq \mathbf{3 / 2}$ then we have

$$
\omega_{p}\left(B_{\delta}, x_{0}, \mathbb{R}_{+}^{N}\right) \leq C_{2} \delta^{\frac{N-1}{p-1}} .
$$

(2) If $\mathbf{3} / \mathbf{2}<\boldsymbol{p} \leq \mathbf{2}$ then we have

$$
C_{1} \delta^{\frac{p+N-3}{2 p-3}} \leq \omega_{p}\left(B_{\delta}, x_{0}, \mathbb{R}_{+}^{N}\right) \leq C_{2} \delta^{\frac{N-1}{p-1}} .
$$

(3) If $\mathbf{2} \leq \boldsymbol{p} \leq \boldsymbol{N}$ then we have

$$
C_{1} \delta^{\frac{N-1}{p-1}} \leq \omega_{p}\left(B_{\delta}, x_{0}, \mathbb{R}_{+}^{N}\right) \leq C_{2} \delta^{\frac{p+N-3}{2 p-3}} .
$$

(4) If $\boldsymbol{p} \geq \boldsymbol{N}$ then we have

$$
C_{1} \delta^{\frac{p+N-3}{2 p-3}} \leq \omega_{p}\left(B_{\delta}, x_{0}, \mathbb{R}_{+}^{N}\right) \leq C_{2} \delta^{\frac{N-1}{p-1}} .
$$

In each case, $C_{1}$ and $C_{2}$ are positive constants only depending on $N$ and $p$.

It should be noted that the estimates in Theorem 1.1 coincide or improve those obtained in [6], depending on the range of $p$. More precisely, let

$$
p^{*}=\frac{8+N+\sqrt{N^{2}+4 N-8}}{6} \text { and } p_{*}=1+\sqrt{N-1} .
$$

Observe that $p^{*}, p_{*} \in[2, N]$. Consider also

$$
\begin{aligned}
\eta^{*} & =\frac{N-p+\sqrt{(N-p)^{2}+4(p+N-3)}}{2(p-1)}, \\
\eta_{*} & =\frac{N-p+\sqrt{(N-p)^{2}+4(2 p-3)(p-1)(N-1)}}{2(2 p-3)} .
\end{aligned}
$$

The exponents of $\delta$ obtained in [6] coincide with the upper estimates in Theorem 1.1 in the range $1<p \leq p^{*}$. In the range $p \geq p^{*}$ DeBlassie and Smits obtain the exponent $\eta^{*}$ in [6]. As for the lower bound, the exponents of $\delta$ in [6] coincide with those of Theorem 1.1 in the range $1<p \leq p_{*}$. In the range $p \geq p_{*}$ the exponent obtained in [6] is $\eta_{*}$. As a consequence of elementary (yet tedious) calculations, it turns out that

$$
\begin{gathered}
\frac{p+N-3}{2 p-3} \geq \eta^{*} \text { if } p^{*} \leq p \leq N, \\
\frac{N-1}{p-1} \geq \eta^{*} \text { if } p \geq N,
\end{gathered}
$$


and

$$
\begin{array}{ll}
\eta_{*} \geq \frac{N-1}{p-1} & \text { if } p_{*} \leq p \leq N, \\
\eta_{*} \geq \frac{p+N-3}{2 p-3} & \text { if } p \geq N,
\end{array}
$$

showing that our estimates improve those of [6].

Our second main result is a purely ODE proof of the existence and uniqueness of a singular quasiradial $p$-harmonic function $r^{k} f(\theta)$ in $\mathbb{R}_{+}^{N}$, where $f(\theta)$ satisfies the full equation

$$
\begin{aligned}
& {\left[(p-1)\left(f^{\prime}\right)^{2}+k^{2} f^{2}\right] f^{\prime \prime}+k[(2 p-3) k+N-p] f\left(f^{\prime}\right)^{2}} \\
& \quad+k^{3}[k(p-1)+N-p] f^{3}+(N-2)\left[\left(f^{\prime}\right)^{2}+k^{2} f^{2}\right] f^{\prime} \cot \theta=0 .
\end{aligned}
$$

Theorem 1.2 Let $1<p<\infty$ and $N \geq 2$. Then there exists a unique $k=k(p, N)<0$ and a function $f:[0, \pi / 2] \rightarrow \mathbb{R}$ satisfying (1.5), (1.6) and (1.7) such that the quasiradial function $r^{k} f(\theta)$ is p-harmonic in $\mathbb{R}_{+}^{N}$.

Our proof of the existence part in Theorem 1.2 is based on topological shooting techniques; it is entirely different from the previous proofs, relying on PDE methods or complicated techniques from harmonic analysis and PDEs on manifolds [12,19,20].

This shooting approach is conceptually simpler and has the added important advantage that it is readily applicable to settings in which solutions do not remain positive and can change sign [21].

Tolksdorff's proof [20] used PDE methods suitable for proving the existence of positive $p$-harmonic functions in cones, which gives also solutions to (1.12) when the cone is the half-space. He also indicated how to get uniqueness from the boundary Harnack inequality. In [19] Tolksdorff's method is extended to cover positive singular solutions.

Our proof of the uniqueness part in Theorem 1.2 is not based on any type of boundary Harnack inequality nor on Martin boundary estimates [15]. Instead we give a direct proof that is a natural extension of our shooting approach, and is motivated by the methods of the landmark papers by Kwong [11] and Coffman [4].

By setting $\alpha(p, N)=-k(p, N)$ we obtain the following consequence of Theorem 1.2 and the results in Sect. 2:

Corollary 1.3 For all $p>1$ and $N \geq 2$ there exists a constant $\alpha(p, N)>0$ such that the p-harmonic measure in $\mathbb{R}_{+}^{N}$ of a ball of radius $0<\delta \leq 1$ in $\mathbb{R}^{N-1}$ satisfies

$$
C_{1} \delta^{\alpha(p, N)} \leq \omega_{p}\left(B_{\delta}, x_{0}, \mathbb{R}_{+}^{N}\right) \leq C_{2} \delta^{\alpha(p, N)} .
$$

Finally, we summarize specific cases where the critical exponent $k(p, N)$ for Eq. (1.17) under conditions (1.5), (1.6), and (1.7) is known.

- $k(2, N)=-(N-1)$. (This corresponds to the case of the Poisson kernel.)

- $k(p, 2)=-\frac{3-p+2 \sqrt{p^{2}-3 p+3}}{3(p-1)}($ see $[2,13])$.

- $k(N, N)=-1$, the conformal case, see [10].

- $k(\infty, N)=-\frac{1}{3}$, see [18].

Note that in the case $p=1, N=2$ equation (1.12) has solutions $f(\theta)=\cos ^{k}(\theta)$ for all $k$. When $k<0$ this solution does not satisfy $f(\pi / 2)=0$, so that Theorem 1.2 does not hold for $p=1$. Apart from such cases, little is known about the exponent $k(p, N)$. 
The organization of the paper is as follows. We show in Sect. 2 how the existence of singular $p$-subharmonic (resp. $p$-superharmonic) functions in the upper half-space implies lower (resp. upper) estimates for $p$-harmonic measure (Theorems 2.1 and 2.2). This section contains standard material based on comparison arguments that we have included for completeness. Section 3 is devoted to the proof of Theorem 3.1, which characterizes the $p$-subharmonicity or $p$-superharmonicity of a specific quasiradial test function, depending on the relation between the parameters $N, k$ and $p$. Finally, In Sect. 4 we provide background on ODE shooting methods and give full details of the uniqueness part of the proof of Theorem 1.2.

\subsection{Remarks}

(1) Theorem 1.1 is a direct consequence of Theorems 2.1, 2.2 and 3.1.

(2) Note that Theorem 1.1 gives the right exponents in the cases $p=2$ and $p=N$.

(3) All of our upper estimates in Theorem 1.1 also hold for spherical caps of the unit ball. However, the lower estimates in the ball do not directly follow from our method.

The research included in this paper originated when the first author was visiting the departments of mathematics at the University of Illinois at Urbana-Champaign and at the University of Pittsburgh. He wishes to thank both institutions for their support.

\section{Estimating $p$-harmonic measures with nonnegative singular quasiradial $p$-super- and $p$-subharmonic functions}

The aim of this section is to show that the existence of quasiradial $p$-superharmonic (resp. $p$-subharmonic) functions $u=r^{k} f(\theta)$ in $\mathbb{R}_{+}^{N}$, where $k<0$ and $f$ satisfies (1.5), (1.6), (1.7), leads to a local upper bound (resp. lower bound) of $p$-harmonic measure on $\partial \Omega$, provided that $\Omega$ satisfies certain geometrical restrictions.

\subsection{Upper bound}

Theorem 2.1 Suppose that there exist $k<0$ and $f:[0, \pi / 2] \rightarrow \mathbb{R}$ satisfying (1.5), (1.6), (1.7) such that the quasiradial function $r^{k} f(\theta)$ is p-superharmonic in $\mathbb{R}_{+}^{N}$. Let $\Omega \subset \mathbb{R}^{N}$ be a convex domain and $x_{0} \in \Omega$. Then there exists $C=C(k, f)>0$ such that for each $\xi \in \partial \Omega$ and any $\delta>0$, we have

$$
\omega_{p}\left(B(\xi, \delta) \cap \partial \Omega, x_{0}, \Omega\right) \leq C\left(\frac{\delta}{\left|x_{0}-\xi\right|}\right)^{|k|} .
$$

Proof From the fact that $0 \leq \omega \leq 1$ and the invariance of $p$-harmonic measure by rescaling, we can assume that $0<\delta<\left|x_{0}-\xi\right|=1$. Finally, from the convexity assumption on $\Omega$ and the rotational invariance of $p$-harmonic measure, we can also assume that $\xi=(0, \ldots 0,2 \delta)$, and that $\Omega \subset\left\{\left(x^{\prime}, x_{N}\right) \in \mathbb{R}^{N}: x_{N}>2 \delta\right\}$. We claim that

$$
\omega_{p}\left(B(\xi, \delta) \cap \partial \Omega, x_{0}, \Omega\right) \leq C \delta^{|k|}
$$

for some $C=C(k, f)>0$.

We put $u(x)=C \delta^{|k|} r^{k} f(\theta)$ where $r=|x|, \theta$ is the azimuth angle, as introduced at the beginning of the section, and the constant $C$ will be chosen later. From the hypothesis, $u$ is $p$-superharmonic in $\mathbb{R}_{+}^{N}$. We put $\omega_{p}(x) \equiv \omega_{p}(B(\xi, \delta) \cap \partial \Omega, x, \Omega)$. We will show that 


$$
\omega_{p} \leq u \text { on } \partial \Omega \text {. }
$$

Observe that $\omega_{p}$ is $p$-harmonic in $\Omega$ and $u$ is $p$-superharmonic in $\Omega$. Then it follows from (2.3) and the comparison principle [9, Thm. 7.6] that $\omega_{p} \leq u$ in $\Omega$. Therefore,

$$
\omega_{p}\left(x_{0}\right) \leq u\left(x_{0}\right) \leq C \delta^{|k|}(1+2 \delta)^{k} \max _{[0, \pi / 2]} f \leq C \delta^{|k|},
$$

and hence (2.2); this completes the proof of the theorem. It remains to show that an appropriate choice of $C$ implies (2.3). We show next that $C=3^{|k|}(f(\pi / 6))^{-1}$ works. Since $\Omega \subset \mathbb{R}_{+}^{N}$, $\omega_{p} \equiv 0$ on $\partial \Omega \backslash B(\xi, \delta)$ and $u$ is positive, we only need to check (2.3) on $B(\xi, \delta) \cap \partial \Omega$. Now let $x \in B(\xi, \delta) \cap \partial \Omega$. If $r=|x|$ and $\theta$ is the azimuth angle of $x$, elementary geometry gives that $r \leq 3 \delta$ and $0 \leq \theta \leq \pi / 6$. By the definition of $u$ and the fact that $f$ is decreasing we have

$$
u(x) \geq C \delta^{|k|}(3 \delta)^{k} f(\pi / 6)=3^{k} f(\pi / 6) C,
$$

so the choice $C=3^{|k|}(f(\pi / 6))^{-1}$ implies that $u(x) \geq 1=\omega_{p}(x)$, and (2.3) follows. This finishes the proof of the theorem.

\subsection{Lower bound}

In this subsection we will restrict our attention to the case where the domain is the half-space $\mathbb{R}_{+}^{N}$.

Theorem 2.2 Suppose that there exist $k<0$ and $f:[0, \pi / 2] \rightarrow \mathbb{R}$ satisfying (1.5), (1.6), (1.7) such that the quasiradial function $r^{k} f(\theta)$ is $p$-subharmonic in $\mathbb{R}_{+}^{N}$. Then there exists a constant $C=C(N, p, k)>0$ such that for any ball $B \subset \mathbb{R}^{N-1}$ of radius $0<\delta \leq 1$ we have

$$
\omega_{p}\left(B, x_{B}, \mathbb{R}_{+}^{N}\right) \geq C \delta^{|k|},
$$

where $x_{B}=\left(a_{B}, 1\right) \in \mathbb{R}_{+}^{N}$ and $a_{B}$ is the center of $B$.

Proof We can assume that $B$ is centered at 0 . Denote by $\omega_{p}($.) the $p$-harmonic measure of $B$ in $\mathbb{R}_{+}^{N}$. Let $\widetilde{B}$ be the $N$-dimensional upper half ball in $\mathbb{R}_{+}^{N}$ centered at 0 and of radius $\delta / 2$. Then it follows from the Carleson estimates for $p$-harmonic measure [1] that there exists $C_{0}=C_{0}(N, p)>0$ such that

$$
\omega_{p}\left(B, x, \mathbb{R}_{+}^{N}\right) \geq C_{0}
$$

whenever $x \in \partial \widetilde{B}$. Now let $\Omega=\mathbb{R}_{+}^{N} \backslash \widetilde{B}$. Then $\partial \Omega=F_{1} \cup F_{2}$ where $F_{1}=\partial \widetilde{B} \cap \mathbb{R}_{+}^{N}$ and $F_{2}=\mathbb{R}^{N-1} \backslash \frac{1}{2} B$. (If $t>0, t B$ stands for the ball concentric to $B$ of radius $t$ times the radius of $B$.)

We define now $v=C_{0} 2^{-|k|} \delta^{|k|} r^{k} f(\theta)$, which is assumed to be $p$-subharmonic in $\mathbb{R}_{+}^{N}$ by the hypothesis. We claim that $v \leq \omega_{p}$ in $\Omega$. Indeed, if $x \in F_{1}$ then, from (2.5) and the fact that $f \leq 1$ and the choice of $C$, we have

$$
v(x) \leq C_{0} \leq \omega_{p}(x) .
$$

If $x \in F_{2}$ then $v(x)=0 \leq \omega_{p}(x)$. Then by the comparison principle we get $v \leq \omega_{p}$ in $\Omega$. Now, evaluating at $x_{0}=(0, \ldots, 0,1)$, we obtain

$$
v\left(x_{0}\right)=C_{0} 2^{-|k|} \delta^{|k|} 1^{k} f(0)=C_{0} 2^{-|k|} \delta^{|k|} \leq \omega_{p}\left(B, x_{0}, \mathbb{R}_{+}^{N}\right),
$$

and (2.4) follows. 


\section{Explicit choices of $p$-subharmonic and $p$-superharmonic singular quasiradial functions}

We note that for a quasiradial function of the form $u=r^{k} f(\theta)$ in $\mathbb{R}_{+}^{N}$ then the sign of $\triangle_{p} u$ coincides with the sign of the differential expression

$$
\begin{aligned}
& {\left[(p-1)\left(f^{\prime}\right)^{2}+k^{2} f^{2}\right] f^{\prime \prime}+k[(2 p-3) k+N-p] f\left(f^{\prime}\right)^{2}} \\
& \quad+k^{3}[k(p-1)+N-p] f^{3}+(N-2)\left[\left(f^{\prime}\right)^{2}+k^{2} f^{2}\right] f^{\prime} \cot \theta .
\end{aligned}
$$

The aim of this section is to seek intervals of the parameter $k<0$ where (3.1) has a definite sign in the interval $[0, \pi / 2]$, and therefore, the corresponding quasiradial function $r^{k} f(\theta)$ is $p$-super- or $p$-subharmonic in $\mathbb{R}_{+}^{N}$. We will show that the choice $f(\theta)=\cos \theta$ produces specific intervals where the sign of $(3.1)$ in $[0, \pi / 2]$ is constant.

It is convenient to set $g=\log f$. Then $f^{\prime}=g^{\prime} e^{g}$ and $f^{\prime \prime}=\left[g^{\prime \prime}+\left(g^{\prime}\right)^{2}\right] e^{g}$. After cancelation of $e^{3 g},(3.1)$ is transformed into

$$
\begin{aligned}
& {\left[(p-1)\left(g^{\prime}\right)^{2}+k^{2}\right]\left[g^{\prime \prime}+\left(g^{\prime}\right)^{2}\right]+k[(2 p-3) k+N-p]\left(g^{\prime}\right)^{2}} \\
& +k^{3}[k(p-1)+N-p]+(N-2)\left[\left(g^{\prime}\right)^{2}+k^{2}\right] g^{\prime} \cot \theta .
\end{aligned}
$$

Now the choice of $f=\cos \theta$ gives $g=\log (\cos \theta), g^{\prime}=-\tan \theta$ and $g^{\prime \prime}=-1-\tan ^{2} \theta$. Making these substitutions in (3.2) we obtain

$$
\begin{gathered}
{\left[(p-1) \tan ^{2} \theta+k^{2}\right](-1)+k[(2 p-3) k+N-p] \tan ^{2} \theta} \\
\quad+k^{3}[k(p-1)+N-p]+(N-2)\left(\tan ^{2} \theta+k^{2}\right)(-1),
\end{gathered}
$$

and, finally, setting $t=\tan ^{2} \theta$ we can write (3.3) as

$$
\Lambda(k, N, p)(t) \equiv \alpha t+\beta k^{2},
$$

where

$$
\begin{aligned}
\alpha & =(2 p-3) k^{2}+(N-p) k+3-N-p \\
\beta & =(p-1) k^{2}+(N-p) k+1-N
\end{aligned}
$$

and $t \geq 0$. We now study the intervals in which the sign of $\Lambda$ is constant in $[0,+\infty)$, in terms of $N, k$ and $p$. It is clear that

$$
\begin{aligned}
& \Lambda \geq 0 \text { in }[0,+\infty) \Leftrightarrow \alpha \geq 0, \beta \geq 0 \text { and that } \\
& \Lambda \leq 0 \text { in }[0,+\infty) \Leftrightarrow \alpha \leq 0, \beta \leq 0 .
\end{aligned}
$$

As a function of the variable $k, \beta(k)$ has zeros $-\frac{N-1}{p-1}$ and 1 . As for the function $\alpha(k)$, unless $p=3 / 2$, its zeros are $-\frac{p+N-3}{2 p-3}$ and 1 . Now consider the graphs of the decreasing functions

$$
f(p)=\frac{p+N-3}{2 p-3} \text { and } g(p)=\frac{N-1}{p-1} .
$$

The graph of $f(p)$ has an asymptotic vertical line at $p=3 / 2$. Furthermore, the graphs only intersect at $p=2$ and $p=N$. In particular, $f<0$ in $(0,3 / 2), f>g$ in $(3 / 2,2), f<g$ in $(2, N)$ and $f>g$ in $(N,+\infty)$.

We collect all this information in the following cases. 
(1) $1<p \leq 3 / 2$. We have $\alpha(k) \leq 0$ and $\beta(k) \leq 0$ in $\left[-\frac{N-1}{p-1}, 0\right)$

(2) $3 / 2<p \leq 2$. We have $\frac{p+N-3}{2 p-3} \geq \frac{N-1}{p-1}$. Thus, we obtain $\alpha(k), \beta(k) \geq 0$ in $\left(-\infty,-\frac{p+N-3}{2 p-3}\right]$ and $\alpha(k), \beta(k) \leq 0$ in $\left[-\frac{N-1}{p-1}, 0\right)$.

(3) $2 \leq p \leq N$. We have $\frac{p+N-3}{2 p-3} \leq \frac{N-1}{p-1}$. Thus, we get $\alpha(k), \beta(k) \geq 0$ in $(-$ $\left.\infty,-\frac{N-1}{p-1}\right]$ and $\alpha(k), \beta(k) \leq 0$ in $\left[-\frac{p+N-3}{2 p-3}, 0\right)$.

(4) $p \geq N$. Again we have $\frac{p+N-3}{2 p-3} \geq \frac{N-1}{p-1}$. Thus, we get $\alpha(k), \beta(k) \geq 0$ in $\left(-\infty,-\frac{p+N-3}{2 p-3}\right]$ and $\alpha(k), \beta(k) \leq 0$ in $\left[-\frac{N-1}{p-1}, 0\right)$.

We have therefore proved the following theorem.

Theorem 3.1 Let $k<0$ and consider the quasiradial function $u=r^{k} \cos \theta$ in $\mathbb{R}_{+}^{N}$.

(1) If $\mathbf{1}<\boldsymbol{p} \leq \mathbf{3 / 2}$, then $u$ is $p$-superharmonic for $k \in\left[-\frac{N-1}{p-1}, 0\right)$.

(2) If $\mathbf{3} / \mathbf{2}<\boldsymbol{p} \leq \mathbf{2}$, then $u$ is $p$-subharmonic for $k \in\left(-\infty,-\frac{p+N-3}{2 p-3}\right]$ and $p$ superharmonic for $k \in\left[-\frac{N-1}{p-1}, 0\right)$.

(3) If $\mathbf{2} \leq \boldsymbol{p} \leq \boldsymbol{N}$, then $u$ is $p$-subharmonicfor $k \in\left(-\infty,-\frac{N-1}{p-1}\right]$ and $p$-superharmonic for $k \in\left[-\frac{p+N-3}{2 p-3}, 0\right)$.

(4) If $\boldsymbol{p} \geq N$, then $u$ is $p$-subharmonic for $k \in\left(-\infty,-\frac{p+N-3}{2 p-3}\right]$ and $p$-superharmonic for $k \in\left[-\frac{N-1}{p-1}, 0\right)$.

Remark Assume, for simplicity, that $N \geq 3$ and let $u=r^{k} \cos \theta$ be as above. Even though we are mainly interested in the case $k<0$, we also record the situation for $k \geq 0$ :

- If $1<p<3 / 2$, then $u$ is $p$-subharmonic for $k \in\left[1, \frac{p+N-3}{3-2 p}\right]$ and $p$-superharmonic for $k \in[0,1]$.

- If $p \geq 3 / 2$, then $u$ is $p$-subharmonic for $k \in[1,+\infty)$ and $p$-superharmonic for $k \in$ $[0,1]$.

Observe that the case $k=1$ corresponds to the function $u(x)=x_{N}$, which is $p$-harmonic for any $p>1$.

\section{ODE theorems: Proof of Theorem 1.2}

Topological shooting methods were developed for the important model equation

$$
\begin{aligned}
& u^{\prime \prime}+\frac{N-1}{r} u^{\prime}+f(u)=0, \\
& u(0)=u_{0}>0 \text { and } u^{\prime}(0)=0 .
\end{aligned}
$$


Bound state solutions satisfy

$$
\lim _{r \rightarrow \infty}\left(u(r), u^{\prime}(r)\right)=(0,0) .
$$

A positive ground state is a solution such that $u(r)>0$ for all $r \geq 0$. Hastings and McLeod [8, pp. 5-6] present a standard two-step approach to prove that the initial value problem is well posed: first, transform (4.1)-(4.2) into the system

$$
\begin{aligned}
& u(r)=u_{0}+\int_{0}^{r} v(\rho) \mathrm{d} \rho, \\
& v(r)=-\frac{1}{r^{N-1}} \int_{0}^{r} \rho^{N-1} f(u(\rho)) d \rho .
\end{aligned}
$$

Second, an application of the method of successive approximations shows that for each $u_{0}>0$ there is an interval $\left[0, r_{1}\right)$ over which a unique solution of (4.4) exists, and this solution depends continuously on $u_{0}$. It also shows (see [3]) that $\frac{\partial u}{\partial u_{0}}$ is continuous. This method applies in general (see [3,7]), including to problem (4.6)-(4.7) studied here. Therefore, problem (4.6)-(4.7) is well posed. Since 1963 the investigation of existence and uniqueness of bound state solutions when

$$
f(u)=\left|u^{p-1}\right| u-u, N>1 \text { and } 1<p<\frac{N+1}{N-1}
$$

has been the central focus of several studies. In 1963 Nehari [17] proved existence of a positive ground state solution when $p=3$. In 1972 Coffman [4] proved uniqueness of the positive ground state when $p=3$. In 1989 Kwong [11] proved uniqueness of the positive ground state in the general case $N>2$ and $1<p<\frac{N+2}{N-2}$. The uniqueness proofs in [4,11] combine the fact that $u(r, \alpha)$ is continuously differentiable function of $r$ and $\alpha$, together with a clever analysis of the equation satisfied by $\frac{\partial u(r, \alpha)}{\partial \alpha}$. In our study of uniqueness we use this same approach to analyze properties of $\frac{\partial y(r, \alpha)}{\partial k}$. Finally, we point out that in 1990 McLeod, Troy and Weissler [16] let $N>1$ and $1<p<\frac{N+1}{N-1}$, and used a topological shooting approach to prove existence of sign changing bound state solutions.

According to (3.1) and (1.7) the initial value problem to consider is

$$
\begin{aligned}
& \left((p-1)\left(y^{\prime}\right)^{2}+k^{2} y^{2}\right) y^{\prime \prime}=((3-2 p) k+p-N) k y\left(y^{\prime}\right)^{2} \\
& \quad+(k(1-p)+p-N) k^{3} y^{3}+(2-N) y^{\prime} \cot (x)\left(\left(y^{\prime}\right)^{2}+k^{2} y^{2}\right), \\
& y(0)=1 \text { and } y^{\prime}(0)=0 .
\end{aligned}
$$

where we have changed the notation $x$ for $\theta$ and $y(x)$ for $f(\theta)$. The goal is to prove that if $N \geq 2$ and $p>1$ there exists $k(p, N)<0$ such that if $k=k(p, N)$ then the solution of (4.6)-(4.7) satisfies

$$
0<y(x)<1 \text { and } y^{\prime}(x)<0 \text { for all } x \in\left(0, \frac{\pi}{2}\right) \text {, and } \lim _{x \rightarrow \frac{\pi}{2}^{-}} y(x)=0 .
$$

Because of the known cases (see Introduction) we always assume $N>2$. It suffices to consider two separate regimes: (a) $1<p<N$, and (b) $p \geq N$.

Theorem 4.1 (a) Let $1<p<N$. There exists a unique $k(p, n) \in\left(-\infty, \frac{N-p}{1-p}\right)$ such that if $k=k(p, n)$ then the solution of (4.6)-(4.7) satisfies (4.8).

(b) Let $p \geq N$. There exists a unique $k(p, n) \in(-\infty, 0)$ such that if $k=k(p, n)$ then the solution of (4.6)-(4.7) satisfies (4.8). 
Our first step in the proof of Theorem 4.1 is to follow Deblassie and Smits [5], and set $y=\exp \left(\int_{0}^{x} H(t) \mathrm{d} t\right)$. Then, we have $y(0)=1$, and substitution into (4.6) gives the first-order equation

$$
\begin{gathered}
\left((p-1) H^{2}+k^{2}\right)\left(H^{\prime}+H^{2}\right)=((3-2 p) k+p-N) k H^{2} \\
+(k(1-p)+p-N) k^{3}+(2-N) H \cot (x)\left(H^{2}+k^{2}\right) .
\end{gathered}
$$

Differentiating and using $y^{\prime}(0)=0$ we get $H(0)=0$. It now follows from (4.9) that property (4.8) holds if $H(x)$ also satisfies

$$
-\infty<H(x)<0 \text { and } H^{\prime}(x)<0 \text { for all } x \in\left(0, \frac{\pi}{2}\right) \text {, and } \lim _{x \rightarrow \frac{\pi}{2}^{-}} H(x)=-\infty .
$$

Thus, Theorem 4.1 follows from

Theorem 4.2 (a) Let $1<p<N$. There exists a unique $k(p, n) \in\left(-\infty, \frac{N-p}{1-p}\right)$ such that if $k=k(p, n)$ then the solution of (4.9) with $H(0)=0$ satisfies (4.10).

(b) Let $p \geq N$. There exists a unique $k(p, n) \in(-\infty, 0)$ such that if $k=k(p, n)$ then the solution of (4.9) with $H(0)=0$ satisfies (4.10).

\subsection{Proof of Theorem 4.2 (existence)}

We make use of a topological shooting technique developed in five steps.

(Step I) First, in Lemma 4.3 we prove, for appropriately chosen $k<0$, that solutions of (4.9) with $H(0)=0$ decrease and satisfy $H(x) \rightarrow-\infty$ at a critical value $x_{k} \in(0, \pi]$.

(Step II) We define two topological shooting sets $S_{1}$ and $S_{2}$.

(a) For $1<p<N$ we set

$$
\begin{aligned}
& S_{1}=\left\{\hat{k}<\frac{N-2}{1-p}: \text { if } \hat{k}<k<\frac{N-2}{1-p}\right. \text { then } \\
& \left.H^{\prime}(x)<0 \text { and }-\infty<H(x)<0 \text { for all } x \in\left(0, \frac{\pi}{2}\right]\right\},
\end{aligned}
$$

(b) and for $p \geq N$ we set

$$
\begin{aligned}
& S_{2}=\left\{\hat{k}<0 \mid \text { if } \hat{k}<k<0 \text { then } H^{\prime}(x)<0\right. \\
& \text { and } \left.-\infty<H(x)<0 \text { for all } x \in\left(0, \frac{\pi}{2}\right]\right\} .
\end{aligned}
$$

Not that it follows from continuity of solutions with respect to $k$ that $S_{1}$ and $S_{2}$ are open.

(Step III) $S_{1}$ and $S_{2}$ are nonempty, open subintervals of $(-\infty, 0)$ (Lemma 4.4).

(Step IV) $S_{1}$ and $S_{2}$ are bounded below (Lemma 4.5)

(StepV) (a) We have that $k(p, N)=\inf S_{1}$ if $1<p<N$ and (b) $k(p, N)=\inf S_{2}$ if $p \geq N$ (Lemma 4.6 ).

Remark Throughout we make use of the fact that (4.9) can be written as

$$
\begin{aligned}
& \left((p-1) H^{2}+k^{2}\right) H^{\prime}=\left(H^{2}+k^{2}\right)^{2}(1-p) \\
& \quad+\left(H^{2}+k^{2}\right)(k(p-N)+(2-N) H \cot (x)) .
\end{aligned}
$$


Lemma 4.3 (a) For $1<p<N$ we have

$$
H^{\prime}(0)=0 \text { if } k=\frac{N-p}{1-p}<0, \text { and } H^{\prime}(0)<0 \text { if } k<\frac{N-p}{1-p},
$$

and for each $k<\frac{N-p}{1-p}$ there exists $x_{k} \in(0, \pi]$ such that

$$
-\infty<H(x)<0 \text { and } H^{\prime}(x)<0 \text { for all } x \in\left(0, x_{k}\right) \text {, and } \lim _{x \rightarrow x_{k}^{-}} H(x)=-\infty \text {. }
$$

(b) For $p \geq N$ we have

$$
H^{\prime}(0)<0 \text { for all } k<0 .
$$

For each $k<0$ there exists $x_{k} \in(0, \pi]$ such that (4.15) holds.

Proof (a) Let $1<p<N$. It follows from (4.9) and $H(0)=0$ that

$$
H^{\prime}(0)=\frac{k(1-p)}{N-1}\left(k-\frac{N-p}{1-p}\right) \text {. }
$$

Since $1<p<N$, we conclude from (4.17) that $H^{\prime}(0)=0$ when $k=\frac{N-p}{1-p}$, and $H^{\prime}(0)<0$ when $k<\frac{N-p}{1-p}$. Next, suppose that there exists $k<\frac{N-p}{1-p}$ and corresponding value $\tilde{x} \in(0, \pi)$ such that

$$
H^{\prime}(x)<0 \text { for all } x \in[0, \tilde{x}), \quad-\infty<H(\tilde{x})<0 \text { and } H^{\prime}(\tilde{x})=0 .
$$

Then

$$
H^{\prime \prime}(\tilde{x}) \geq 0 .
$$

It follows from a differentiation of (4.9) and (4.18) that

$$
\left((p-1) H^{2}(\tilde{x})+k^{2}\right) H^{\prime \prime}(\tilde{x})=(N-2) \csc ^{2}(\tilde{x}) H(\tilde{x})\left(H^{2}(\tilde{x})+k^{2}\right)<0,
$$

contradicting (4.19). We conclude that $-\infty<H(x)<0$ and $H^{\prime}(x)<0$ for $x \in(0, \pi)$ as long as the solution exists. Thus, for each $k<\frac{N-p}{1-p}$ we let $\left[0, x_{k}\right)$ denote the maximal subinterval of $[0, \pi]$ over which the solution of (4.9) exists. We need to prove that

$$
\lim _{x \rightarrow x_{k}^{-}} H(x)=-\infty \text { for all } k<\frac{N-p}{1-p} .
$$

First, suppose there exists $k<\frac{N-p}{1-p}$ such that $x_{k} \geq \pi$. We need to prove that

$$
\lim _{x \rightarrow \pi^{-}} H(x)=-\infty .
$$

Suppose, however, that (4.22) does not hold, and that

$$
-\infty<H(x)<0, \quad H^{\prime}(x)<0 \text { for all } x \in(0, \pi) \text {, and }-\infty<H(\pi)<0 .
$$

Dividing (4.13) by $(p-1) H^{2}+k^{2}$, we conclude, since $1-p<0$ and $k p<0$, that

$$
H^{\prime} \leq \frac{H^{2}+k^{2}}{(p-1) H^{2}+k^{2}}(-k N+(2-N) H \cot (x)) \text { for all } x \in(0, \pi) \text {. }
$$


We need upper bounds on the terms on the right side of (4.24). For this define

$$
L=\min \left\{1, \frac{1}{p-1}\right\} \text {. }
$$

It is easily verified that

$$
L \leq \frac{H^{2}+k^{2}}{(p-1) H^{2}+k^{2}} \leq \frac{p}{p-1} \quad \forall H<0 \text { and } k<0 .
$$

We focus on the interval $\frac{\pi}{2} \leq x<\pi$ where $-\infty<\cot (x) \leq 0$. First, define

$$
M_{1}=-\frac{k N p}{p-1}>0 \text { and } M_{2}=(2-N) H\left(\frac{\pi}{2}\right) L>0 .
$$

Combining (4.24) with (4.25), (4.26), and (4.27), we obtain

$$
H^{\prime} \leq M_{1}+M_{2} \cot (x), \quad \frac{\pi}{2} \leq x<\pi .
$$

Integrating (4.28), we conclude that

$$
H(x) \leq H\left(\frac{\pi}{2}\right)+M_{1}\left(x-\frac{\pi}{2}\right)+M_{2} \ln (\sin (x)), \quad \frac{\pi}{2} \leq x<\pi .
$$

Since $M_{2}>0$, it follows from (4.29) that $H(x) \rightarrow-\infty$ as $x \rightarrow \pi^{-}$, which contradicts (4.23). Thus, it must be the case that (4.22) holds if $x_{k}=\pi$. Finally, we need to consider the possibility that there exists $k<\frac{N-p}{1-p}$ such that $0<x_{k}<\pi$ and (4.21) does not hold. Then

$$
-\infty<H(x)<0 \text { and } H^{\prime}(x)<0 \text { for all } x \in\left(0, x_{k}\right)
$$

and

$$
-\infty<H\left(x_{k}\right)<0 .
$$

Then $(p-1) H^{2}\left(x_{k}\right)+k^{2}>0$ is bounded and all terms on the right side of (4.13) are nonzero and bounded. Thus, the solution can be uniquely continued past $x_{k}$, which contradicts the definition of $x_{k}$. This completes the proof of property (4.21) and part (a). The proof of part (b) is essentially the same and is omitted for the sake of brevity.

Lemma 4.4 (a) For $1<p<N$ there exists $k_{1}<\frac{N-p}{1-p}$ such that

$$
k \in S_{1} \text { for all } k \in\left(k_{1}, \frac{N-p}{1-p}\right) .
$$

(b) For $p \geq N$ there exists $k_{2}<0$ such that

$$
k \in S_{2} \text { for all } k \in\left(k_{2}, 0\right) .
$$

Proof (a) For $0 \leq x \leq \frac{\pi}{2}$, as long as $H^{\prime}(x)<0$ and $-\infty<H(x) \leq 0$ we conclude that $(2-N) H(x) \cot (x) \geq 0$, ; hence, (4.13) reduces to

$$
\left((p-1) H^{2}+k^{2}\right) H^{\prime} \geq\left(H^{2}+k^{2}\right)\left((1-p)\left(H^{2}+k^{2}\right)+k(p-N)\right) .
$$

Next, divide (4.34) by $(p-1) H^{2}+k^{2}$, rearrange terms and obtain

$$
H^{\prime} \geq \frac{\left(H^{2}+k^{2}\right)(1-p)}{(p-1) H^{2}+k^{2}}\left(H^{2}+k\left(k-\frac{N-p}{1-p}\right)\right) \text {. }
$$


Note that

$$
k\left(k-\frac{N-p}{1-p}\right)>0 \text { for all } k<\frac{N-p}{1-p}<0 .
$$

Thus, when $k<\frac{N-p}{1-p}<0$, we combine inequality (4.26) with (4.35) and (4.36), and conclude that

$$
H^{\prime} \geq-p\left(H^{2}+k\left(k-\frac{N-p}{1-p}\right)\right)
$$

for $x \in\left[0, \frac{\pi}{2}\right]$ as long as $-\infty<H(x) \leq 0$ and $H^{\prime}(x)<0$. An integration of (4.37) gives

$$
H(x) \geq-\sqrt{k\left(k-\frac{N-p}{1-p}\right)} \tan \left(x p \sqrt{k\left(k-\frac{N-p}{1-p}\right)}\right)>-\infty
$$

for $x \in\left[0, \frac{\pi}{2}\right]$ as long as $-\infty<H(x) \leq 0$ and $H^{\prime}(x)<0$. Finally, let $k_{1}<\frac{N-p}{1-p}$ such that

$$
p \sqrt{k\left(k-\frac{N-p}{1-p}\right)}<1 \quad \forall k \in\left(k_{1}, \frac{N-p}{1-p}\right) .
$$

Thus, when $k \in\left(k_{1}, \frac{N-p}{1-p}\right)$, we conclude from (4.38), (4.39) and Lemma 4.3 that

$$
H(x) \geq-\sqrt{k\left(k-\frac{N-p}{1-p}\right)} \tan \left(\sqrt{k\left(k-\frac{N-p}{1-p}\right)} p x\right)>-\infty \forall x \in\left[0, \frac{\pi}{2}\right],
$$

and that

$$
H^{\prime}(x)<0 \quad \forall x \in\left[0, \frac{\pi}{2}\right]
$$

It follows from (4.40) and (4.41) that $k \in S_{1}$ for all $k \in\left(k_{1}, \frac{N-p}{1-p}\right)$. This completes the proof of part (a).

Part (b). There are two small adjustments to make. First, because $\frac{N-p}{1-p} \geq 0$, we replace (4.36) with

$$
k\left(k-\frac{N-p}{1-p}\right)>0 \text { for all } k<0
$$

Second, in place of choosing $k_{1}$ to satisfy (4.39), we let $k_{2}<0$ be chosen such that

$$
p \sqrt{k\left(k-\frac{N-p}{1-p}\right)}<1 \text { for all } k \in\left(k_{2}, 0\right) .
$$

With these adjustments the proof of (b) is the same as the proof of (a), and we omit the details for the sake of brevity.

Lemma 4.5 (a) For $1<p<N$ there is a value $k_{3}<\frac{N-p}{1-p}<0$ such that $0<x_{k_{3}} \leq \frac{\pi}{2}$.

(b) For $p \geq N$ there is a value $k_{4}<0$ such that $0<x_{k_{4}} \leq \frac{\pi}{2}$. 
Proof (a) We assume, for contradiction, that

$$
x_{k}>\frac{\pi}{2} \text { for all } k<\frac{N-p}{1-p} .
$$

Throughout we focus on the interval $\left[\frac{\pi}{4}, \frac{\pi}{2}\right]$. First, we observe that

$$
0 \leq \cot (x) \leq 1 \quad \text { and } 0<\frac{k^{2}}{(p-1) H^{2}+k^{2}} \leq 1 \quad \forall x \in\left[\frac{\pi}{4}, \frac{\pi}{2}\right] .
$$

When $N>2, p>1, \frac{\pi}{4} \leq x \leq \frac{\pi}{2}, H<0$ and $k<0$ we conclude that

$$
0<\frac{(2-N) H \cot (x)}{(p-1) H^{2}+k^{2}} \leq\left(\frac{2-N}{2 k \sqrt{p-1}}\right)\left(\frac{2 k \sqrt{p-1} H}{(p-1) H^{2}+k^{2}}\right) \leq \frac{2-N}{2 k \sqrt{p-1}},
$$

since $(\sqrt{(p-1)} H-k)^{2} \geq 0$. Next, divide (4.13) by $(p-1) H^{2}+k^{2}$ and get

$$
\begin{aligned}
H^{\prime}= & \left(H^{2}+k^{2}\right)\left(\left(\frac{(1-p)\left(H^{2}+k^{2}\right)}{(p-1) H^{2}+k^{2}}\right)+\left(\frac{p-N}{k}\right)\left(\frac{k^{2}}{(p-1) H^{2}+k^{2}}\right)\right) \\
& +\left(H^{2}+k^{2}\right)\left(\frac{(2-N) H \cot (x)}{(p-1) H^{2}+k^{2}}\right) .
\end{aligned}
$$

Combining (4.26), (4.44), (4.46) and(4.47), we conclude that

$$
H^{\prime} \leq\left(H^{2}+k^{2}\right)\left((1-p) L+\frac{(p-N)}{k}+\frac{2-N}{2 k \sqrt{p-1}}\right) \text {. }
$$

Now let $\tilde{k}<\frac{N-2}{1-p}<0$ such that

$$
\frac{(1-p) L}{2}+\frac{(p-N)}{k}+\frac{2-N}{2 k \sqrt{p-1}}<0 \text { for all } k<\tilde{k} .
$$

It follows from (4.48) and (4.49) that

$$
H^{\prime} \leq \frac{(1-p) L}{2}\left(H^{2}+k^{2}\right) \text { for all } x \in\left[\frac{\pi}{4}, \frac{\pi}{2}\right] \text { and } k<\tilde{k} .
$$

Integrating (4.50) from $\frac{\pi}{4}$ to $x$ gives

$$
\tan ^{-1}\left(\frac{H(x)}{k}\right) \geq \tan ^{-1}\left(\frac{H(\pi / 4)}{k}\right)+\frac{L k(1-p)}{2}\left(x-\frac{\pi}{4}\right) \text { for all } x \in\left[\frac{\pi}{4}, \frac{\pi}{2}\right] .
$$

Assumption (4.44), and the fact that $H(x)$ is decreasing on $\left[0, x_{k}\right]$, imply that

$$
\frac{\pi}{2}>\tan ^{-1}\left(\frac{H(x)}{k}\right) \geq \tan ^{-1}\left[\frac{H(\pi / 4)}{k}\right)>0 \text { for all } x \in\left[\frac{\pi}{4}, \frac{\pi}{2}\right] \text { and } k<\tilde{k} \text {. }
$$

Thus, when $x=\frac{\pi}{2}$, it follows from (4.51) and (4.52) that

$$
\frac{\pi}{2}>\tan ^{-1}\left(\frac{H(\pi / 2)}{k}\right)>\frac{L k(1-p)}{8} \pi>\frac{\pi}{2}, \text { for all } k<\min \left\{\tilde{k}, \frac{4}{L(1-p)}\right\},
$$


a contradiction. Therefore, we conclude that (4.44) does not hold, and that there exists $k_{3}<\frac{N-2}{1-p}$ such that $x_{k_{3}} \leq \frac{\pi}{2}$. The proof of part (b) is essentially the same as the proof of (a), and is omitted for the sake of brevity.

Lemma 4.6 (a) For $1<p<N$ we have

$$
k(p, N)=\inf S_{1} .
$$

(b) For $p \geq N$ we have

$$
k(p, N)=\inf S_{2} .
$$

Proof (a) It follows from the definition of $S_{1}$, Lemma 4.4 and Lemma 4.5 that $k_{*}=\inf S_{1}>$ $-\infty$. Let $H_{*}(x)$ denote the solution of (4.9) with $k=k_{*}$. When $k=k_{*}$ Lemma 4.3 implies that $x_{k_{*}} \in(0, \pi]$ exists such that

$-\infty<H_{*}(x)<0$ and $H_{*}^{\prime}(x)<0$ for all $x \in\left(0, x_{k_{*}}\right)$, and $\lim _{x \rightarrow x_{k_{*}}^{-}} H_{*}(x)=-\infty$.

Our goal is to show that $x_{k_{*}}=\frac{\pi}{2}$, from which we conclude that $k(p, N)=k_{*}=\inf S_{1}$. For contradiction we assume that $x_{k_{*}} \neq \frac{\pi}{2}$. Then $x_{k_{*}} \in\left(0, \frac{\pi}{2}\right) \cup\left(\frac{\pi}{2}, \pi\right]$. Suppose that $x_{k_{*}} \in\left(\frac{\pi}{2}, \pi\right]$. Then $k_{*} \in S_{1}$ and we conclude from the definition of $S_{1}$, Lemma 4.3 and continuity of solutions with respect to $k$ over the compact interval $\left[0, \frac{\pi}{2}\right]$ that, if $k_{*}-k>0$ is sufficiently small, then

$$
-\infty<H(x)<0 \text { and } H^{\prime}(x)<0 \text { for all } x \in\left(0, \frac{\pi}{2}\right] \text {. }
$$

Thus, $k \in S_{1}$ if $k_{*}-k>0$ is sufficiently small, which contradicts the definition of $k_{*}$. It remains to consider the second possibility that $0<x_{k_{*}}<\frac{\pi}{2}$. We define

$$
\delta=\frac{\pi}{2}-x_{k_{*}}>0
$$

We restrict our attention to the interval $\left[\frac{x_{k_{*}}}{2}, \frac{\pi}{2}\right]$ where

$$
0 \leq \cot (x) \leq R=\cot \left(\frac{x_{k_{*}}}{2}\right) \text { for all } x \in\left[\frac{x_{k_{*}}}{2}, \frac{\pi}{2}\right] \text {. }
$$

It follows from (4.56) that there is an $\tilde{x} \in\left(\frac{x_{k_{*}}}{2}, x_{k_{*}}\right)$ such that

$$
\begin{aligned}
& -\infty<H_{*}(\tilde{x})<-1, \\
& 0<\left|\frac{1}{k_{*}}\left(\frac{\pi}{2}-\tan ^{-1}\left(\frac{H_{*}(\tilde{x})}{k}\right)\right)\right|<\frac{\delta}{20},
\end{aligned}
$$

and

$$
0<\frac{\pi}{2}\left(\frac{\left|k_{*}\right||p-N|+k_{*}^{2}|2-p|+(N-2) R}{(p-1)\left|H_{*}(\tilde{x})\right|}\right)<\frac{\delta}{20} .
$$

We conclude from (4.60)-(4.61)-(4.62), and continuity of solutions with respect to $k$ over the interval $[0, \tilde{x}]$ that if $k-k_{*}>0$ is sufficiently small then $H(x)$ satisfies

$$
\begin{aligned}
& -\infty<H(\tilde{x})<-1, \\
& 0<\left|\frac{1}{k}\left(\frac{\pi}{2}-\tan ^{-1}\left(\frac{H(\tilde{x})}{k}\right)\right)\right|<\frac{\delta}{10},
\end{aligned}
$$


and

$$
0<\frac{\pi}{2}\left(\frac{\left|k_{*}\right||p-N|+k_{*}^{2}|2-p|+(N-2) R}{(p-1)|H(\tilde{x})|}\right)<\frac{\delta}{10} .
$$

Next, recall from Lemma 4.4 that

$$
x_{k}>\frac{\pi}{2} \quad \forall k \in S_{1}
$$

It follows from (4.66), and the fact that $H^{\prime}(x)<0$ for all $x \in\left(0, x_{k_{*}}\right)$, that (4.63)-(4.64)(4.65) can be extended to the entire interval $\left[\tilde{x}, \frac{\pi}{2}\right]$, that is,

$$
\begin{aligned}
& -\infty<H(x)<-1 \text { for all } x \in\left[\tilde{x}, \frac{\pi}{2}\right] \\
& 0<\left|\frac{1}{k}\left(\frac{\pi}{2}-\tan ^{-1}\left(\frac{H(x)}{k}\right)\right)\right|<\frac{\delta}{10} \text { for all } x \in\left[\tilde{x}, \frac{\pi}{2}\right],
\end{aligned}
$$

and

$$
0<\frac{\pi}{2}\left(\frac{\left|k_{*}\right||p-N|+k_{*}^{2}|2-p|+(N-2) R}{(p-1)|H(x)|}\right)<\frac{\delta}{10} \text { for all } x \in\left[\tilde{x}, \frac{\pi}{2}\right],
$$

when $k-k_{*}>0$ is sufficiently small. In order to make use of properties (4.67)-(4.68)-(4.69) we first write (4.13) as

$$
\begin{aligned}
& \left((p-1) H^{2}+k^{2}\right) H^{\prime}=\left(H^{2}+k^{2}\right)\left((1-p) H^{2}-k^{2}\right) \\
& \quad+\left(H^{2}+k^{2}\right)\left(k^{2}(2-p)+k(p-N)+(2-N) H \cot (x)\right) .
\end{aligned}
$$

Dividing (4.70) by $(p-1) H^{2}+k^{2}$ gives

$$
H^{\prime}=\left(H^{2}+k^{2}\right)(-1+F(x)),
$$

where

$$
F(x)=\frac{k^{2}(2-p)+k(p-N)+(2-N) H(x) \cot (x)}{(p-1) H^{2}(x)+k^{2}} .
$$

Finally, we divide (4.71) by $H^{2}+k^{2}$ and obtain

$$
\frac{d H}{H^{2}+k^{2}}=(-1+F) \mathrm{d} x
$$

Integrating (4.73) from $\tilde{x}$ to $x$ gives

$$
\frac{1}{k}\left(\tan ^{-1}\left(\frac{H(x)}{k}\right)-\tan ^{-1}\left(\frac{H(\tilde{x})}{k}\right)\right)=\tilde{x}-x+\int_{\tilde{x}}^{x} F(t) \mathrm{d} t, \text { for all } x \in\left[\tilde{x}, \frac{\pi}{2}\right] .
$$

Setting $x=\frac{\pi}{2}$ in (4.74), we obtain

$$
\frac{\pi}{2}-\tilde{x}=\frac{1}{k}\left(\tan ^{-1}\left(\frac{H(\tilde{x})}{k}\right)-\frac{\pi}{2}\right)+\frac{1}{k}\left(\frac{\pi}{2}-\tan ^{-1}\left(\frac{H(\pi / 2)}{k}\right)\right)+\int_{\tilde{x}}^{\pi / 2} F(t) \mathrm{d} t .
$$


We need upper an bound on the right side of (4.75). First, it follows from (4.68) that

$$
\begin{aligned}
& \left|\frac{1}{k}\left(\tan ^{-1}\left(\frac{H(\tilde{x})}{k}\right)-\frac{\pi}{2}\right)+\frac{1}{k}\left(\frac{\pi}{2}-\tan ^{-1}\left(\frac{H(\pi / 2)}{k}\right)\right)\right| \\
& \leq\left|\frac{1}{k}\left(\tan ^{-1}\left(\frac{H(\tilde{x})}{k}\right)-\frac{\pi}{2}\right)\right|+\left|\frac{1}{k}\left(\frac{\pi}{2}-\tan ^{-1}\left(\frac{H(\pi / 2)}{k}\right)\right)\right|<\frac{\delta}{5}
\end{aligned}
$$

when $k-k_{*}>0$ is sufficiently small and $\tilde{x} \leq x \leq \frac{\pi}{2}$. Next, we note that $|k| \leq\left|k_{*}\right|$ and $|k|^{2} \leq\left|k_{*}\right|^{2}$ when $k_{*}<k<\frac{N-p}{1-p}<0$. From these inequalities, property (4.59) and the definition of $F$ given in (4.72) we obtain

$$
\begin{aligned}
& |F(x)| \leq \frac{\left|k_{*}\right|^{2}|2-p|+\left|k_{*}\right||p-N|+|2-N||H(x)| R}{(p-1) H^{2}(x)+k^{2}} \\
& \quad=\frac{\left|k_{*}\right|^{2}|2-p|+\left|k_{*}\right||p-N|}{(p-1) H^{2}(x)+k^{2}}+\frac{|2-N||H(x)| R}{(p-1) H^{2}(x)+k^{2}} \text { for all } x \in\left[\tilde{x}, \frac{\pi}{2}\right] .
\end{aligned}
$$

It follows from (4.67) and the fact that $H^{\prime}(x)<0$ for all $x \in\left[\tilde{x}, \frac{\pi}{2}\right]$, that

$$
H^{2}(x) \geq|H(x)| \geq|H(\tilde{x})| \text { for all } x \in\left[\tilde{x}, \frac{\pi}{2}\right] .
$$

We conclude from (4.78) that

$$
\frac{1}{(p-1) H^{2}(x)+k^{2}} \leq \frac{1}{(p-1)|H(x)|} \leq \frac{1}{(p-1)|H(\tilde{x})|} \text { for all } x \in\left[\tilde{x}, \frac{\pi}{2}\right]
$$

and

$$
\frac{|H(x)|}{(p-1) H^{2}(x)+k^{2}} \leq \frac{|H(x)|}{(p-1)\left|H^{2}(x)\right|} \leq \frac{1}{(p-1)|H(x)|} \leq \frac{1}{(p-1)|H(\tilde{x})|}
$$

for all $x \in\left[\tilde{x}, \frac{\pi}{2}\right]$. Next, we combine (4.77), (4.79)and (4.80), and obtain

$$
|F(x)| \leq \frac{\left|k_{*}\right|^{2}|2-p|+\left|k_{*}\right||p-N|+|2-N| R}{(p-1)|H(\tilde{x})|} \text { for all } x \in\left[\tilde{x}, \frac{\pi}{2}\right] .
$$

It follows from (4.64) and (4.81), and the fact that $\left|\int_{\tilde{x}}^{\pi / 2} F(t) \mathrm{d} t\right| \leq \int_{\tilde{x}}^{\pi / 2}|F(t)| \mathrm{d} t$ that

$$
\left|\int_{\tilde{x}}^{\pi / 2} F(t) \mathrm{d} t\right| \leq \frac{\pi}{2}\left(\frac{\left|k_{*}\right|^{2}|2-p|+\left|k_{*}\right||p-N|+|2-N| R}{(p-1)|H(\tilde{x})|}\right)<\frac{\delta}{10} .
$$

Finally, we substitute (4.58), (4.76) and (4.82) into equation (4.75), and obtain

$$
\delta=\frac{\pi}{2}-\tilde{x} \leq \frac{\delta}{5}+\frac{\delta}{10}=\frac{3}{10} \delta,
$$

which is clearly a contradiction 


\subsection{Proof of Theorem 4.2 (uniqueness)}

Our proof, which relies on an analysis of the equations satisfied by $U=\frac{H(x)}{k}$ and $\frac{\partial U}{\partial k}$, is divided into five basic steps:

(Step I) First, in Lemma 4.7 we prove that solutions of (4.6)-(4.7) must satisfy the fundamentally important property $-\infty<y^{\prime}(\pi / 2)<0$.

(Step II) In Lemma 4.8 we analyze the equation satisfied by $U(x)=\frac{H(x)}{k}$, and prove key properties of $U(x)$, namely

$$
U^{\prime}(x)>0 \quad \forall x \in\left[0, x_{k}\right) \text { and } \lim _{x \rightarrow x_{k}^{-}} U(x)=\infty,
$$

for each negative $k$ in an appropriately chosen range. The function $U(x)$ satisfies the equation

$$
\left((p-1) U^{2}+1\right) U^{\prime}=\left(U^{2}+1\right)^{2} k(1-p)+\left(U^{2}+1\right)(p-N+(2-N) U \cot (x)) .
$$

(Step III) In Lemma 4.9 we determine the behavior of $U$ as $k$ varies. Where it is appropriate we write $U(x, k)$ to emphasize the fact that $U$ depends on both $x$ and $k$. To determine the behavior of $U(x, k)$ we follow the approach by Coffman [4] and Kwong [11], who proved uniqueness of positive ground state solutions of

$$
u^{\prime \prime}+\frac{N-1}{r} u^{\prime}+u^{p}-u=0, \quad u(0)=\alpha>0 \text { and } u^{\prime}(0)=0,
$$

where $N>1$ and $1<p<\frac{N+1}{N-1}$. Their proofs of uniqueness combine the fact that $u(r, \alpha)$ is continuously differentiable function of $r$ and $\alpha$, together with a clever analysis of $\frac{\partial u(r, \alpha)}{\partial \alpha}$. We also follow their approach and combine these differentiability properties together with an analysis of the equation satisfied by $\frac{\partial U(x, k)}{\partial k}$.

In Lemma 4.9 and Lemma 4.10 we analyze the behavior of solutions of the equation satisfied by $\frac{\partial U(x, k)}{\partial k}$, and prove that

$$
\frac{\partial U(x, k)}{\partial k}<0 \text { for all } x \in\left[0, x_{k}\right),
$$

where $k<0$ is in an appropriately chosen range.

(Step IV) We assume, for contradiction, that there are two negative $k$ values, say $k_{1}<k_{2}<0$, and corresponding solutions $y_{1}$ and $y_{2}$ of (4.6)-(4.7) which satisfy property (4.8). The key to obtaining a contradiction of this assumption is to extend (4.87) to

$$
\frac{\partial U(x, k)}{\partial k}<0 \text { for all } x \in\left[0, x_{k}\right) \text { and for all } k \in\left[k_{1}, k_{2}\right] .
$$

This is the goal of Lemma 4.10.

(Step V) We show how to make use of the results described in (I)-(IV) to obtain a contradiction of the assumption that two solutions exist.

Lemma 4.7 (a) For $1<p<N$ suppose that $k<\frac{N-p}{1-p}<0$ exists such that the solution of (4.6)-(4.7) satisfies property (4.8). Then we have

$$
-\infty<y^{\prime}\left(\frac{\pi}{2}\right)<0 \text {. }
$$

(b) For $p \geq N$ suppose that $k<0$ exists such that the solution of (4.6)-(4.7) satisfies property (4.8). Then the solution also satisfies property (4.89). 
Proof (a) Suppose that $k<\frac{N-p}{1-p}<0$ exists such that the solution of (4.6)-(4.7) satisfies (4.8). Then it is easily verified that

$$
0 \leq \frac{(p-1)\left(y^{\prime}\right)^{2}}{(p-1)\left(y^{\prime}\right)^{2}+k^{2} y^{2}} \leq 1 \text { and } 0 \leq \frac{k^{2} y^{2}}{(p-1)\left(y^{\prime}\right)^{2}+k^{2} y^{2}} \leq 1 \text { for all } \in\left[0, x_{k}\right) .
$$

Next, we observe that

$$
(1-p) k+p-N=(1-p)\left(k-\frac{N-p}{1-p}\right)>0 .
$$

Combining (4.6), (4.90), (4.91), and the fact the $0 \leq y \leq 1$ for all $x \in\left[0, \frac{\pi}{2}\right]$, gives

$$
y^{\prime \prime} \geq-\frac{2 p k^{2}}{p-1}-k^{2}(p-1) \quad \forall x \in\left[0, \frac{\pi}{2}\right] .
$$

An integration of (4.92) gives

$$
y^{\prime} \geq-\left(\frac{2 p k^{2}}{p-1}+k^{2}(p-1)\right) x \quad \forall x \in\left[0, \frac{\pi}{2}\right] .
$$

From (4.93) it follows that

$$
y^{\prime}\left(\frac{\pi}{2}\right) \geq-\left(\frac{2 p k^{2}}{p-1}+k^{2}(p-1)\right) \frac{\pi}{2}>-\infty .
$$

It remains to prove that $y^{\prime}\left(\frac{\pi}{2}\right)<0$. It follows from the first inequality in (4.8) that $y^{\prime}\left(\frac{\pi}{2}\right) \leq 0$. Suppose that $y^{\prime}\left(\frac{\pi}{2}\right)=0$. Then it follows from the fact that $y\left(\frac{\pi}{2}\right)=0$, and uniqueness of solutions that $y(x)=0$ for all $x \in\left[0, \frac{\pi}{2}\right]$, contradicting the first inequality in (4.8). This proves (4.89) $1<p<N$. The proof of (4.89) $p \geq N$ is essentially the same and we omit the details.

Lemma 4.8 (a) For $1<p<N$ and each $k<\frac{N-p}{1-p}<0$ the function $U(x)$ satisfies

$$
U^{\prime}(x)>0 \text { for all } x \in\left[0, x_{k}\right) \text { and } \lim _{x \rightarrow x_{k}^{-}} U(x)=\infty .
$$

(b) For $p \geq N$ and for each $k<0$ the function $U(x)$ satisfies (4.95).

Proof Proof. (a) First, it follows from $H(0)=0$ that

$$
U(0)=0 \text { for all } k<\frac{N-p}{1-p}<0 .
$$

Lemma 4.3 shows that, for each $k<\frac{N-p}{1-p}<0$, there is an $x_{k} \in(0, \pi]$ such that

$$
-\infty<H(x)<0 \text { for all } x \in\left(0, x_{k}\right), \text { and } \lim _{x \rightarrow x_{k}^{-}} H(x)=-\infty .
$$

We conclude that

$$
0<U(x)<\infty \text { for all } x \in\left(0, x_{k}\right), \text { and } \lim _{x \rightarrow x_{k}^{-}} U(x)=\infty .
$$


It remains to prove that $U^{\prime}(x)>0$ for all $x \in\left[0, x_{k}\right)$. First, we conclude from (4.91), (4.85) and initial condition (4.96) that

$$
U^{\prime}(0)=\frac{(1-p) k+p-N}{N-1}>0 \text { for all } k<\frac{N-p}{1-p}<0 .
$$

Suppose, for contradiction, that for some $k<\frac{N-p}{1-p}<0$, there exists an $x_{k}^{*} \in\left(0, x_{k}\right)$ such that

$$
U^{\prime}(x)>0 \text { for all } x \in\left(0, x_{k}^{*}\right) \text { and } U^{\prime}\left(x_{k}^{*}\right)=0 .
$$

We conclude from (4.100) that

$$
U^{\prime \prime}\left(x_{k}^{*}\right) \leq 0 .
$$

However, it follows from a differentiation of (4.85) that

$$
U^{\prime \prime}\left(x_{k}^{*}\right)=\left(\frac{U^{2}\left(x_{k}^{*}\right)+1}{(p-1) U^{2}\left(x_{k}^{*}\right)+1}\right)(N-2)\left(\csc ^{2} x_{k}^{*}\right)>0,
$$

contradicting (4.101). This completes the proof of part (a).

Proof of (b). As before, we have

$$
U(0)=0 \text { for all } k<0 .
$$

Since $p \geq N$, we conclude from (4.99) that

$$
U^{\prime}(0)=\frac{(1-p) k+p-N}{N-1}>0 \text { for all } k<0 .
$$

The remaining details of the proof of (b) are now the same as the proof of part (a), and are omitted for the sake of brevity.

Lemma 4.9 (a) For $1<p<N$ and $k<\frac{N-p}{1-p}<0$ we have

$$
\frac{\partial U(x, k)}{\partial k}<0 \text { for all } x \in\left(0, x_{k}\right) \text {. }
$$

(b) For $p \geq N$ and $k<0$ property (4.105) holds.

Proof. (a) Following Coffman [4] and Kwong [11], we set

$$
W=\frac{\partial U(x, k)}{\partial k},
$$

take the partial derivative of (4.85) and (4.96) and with respect to $k$, and obtain

$$
W^{\prime}=Q W+(1-p) \frac{\left(U^{2}+1\right)^{2}}{(p-1) U^{2}+1}, \quad W(0)=0,
$$

where

$$
Q=\frac{(1-p)\left(2 U U^{\prime}+4\left(U^{3}+U\right) k\right)+2 U(p-N)+\left(3 U^{2}+1\right)(2-N) \cot (x)}{(p-1) U^{2}+1} .
$$


An integration of (4.107) gives

$$
W=e^{\int_{0}^{x} Q(t) \mathrm{d} t} \int_{0}^{x}(1-p) \frac{\left(U^{2}(t)+1\right)^{2}}{(p-1) U^{2}+1} e^{-\int_{0}^{t} Q(u) \mathrm{d} u} \mathrm{~d} t<0 \text { for all } x \in\left(0, x_{k}\right) .
$$

This completes the proof of (a). The proof of (b) is the same as (a), and we omit the details for the sake of brevity.

Lemma 4.10 (a) Let $N>2$ and $1<p<N$, and assume that there exist $k_{1}<k_{2}<\frac{N-p}{1-p}$, and corresponding solutions $y_{1}$ and $y_{2}$ of (4.6)-(4.7) which satisfy (4.8). Then

$$
\frac{\partial U(x, k)}{\partial k}<0 \text { for all } \in\left[k_{1}, k_{2}\right] \text { and for all } x \in\left(0, \frac{\pi}{2}\right) \text {. }
$$

(b) For $p \geq N$ assume that there exist $k_{1}<k_{2}<0$, and corresponding solutions $y_{1}$ and $y_{2}$ of (4.6)-(4.7) which satisfy (4.8). Then property (4.110) holds.

Proof (a) The first step in proving (4.110) is to define

$$
S_{3}=\left\{\hat{x} \in\left(0, \frac{\pi}{2}\right) \mid U(x, k) \text { exists for all } k \in\left[k_{1}, k_{2}\right] \text { and for all } x \in(0, \hat{x}]\right\} .
$$

We need to prove that

$$
\mathrm{S}_{3} \text { is open, } S_{3} \neq \phi \text { and } S_{3}=\left(0, \frac{\pi}{2}\right) \text {. }
$$

First, we show that $S_{3}$ is open. Let $\bar{x} \in S_{3}$. We need to prove that there exists $\bar{\epsilon}>0$ such that

$$
[\bar{x}, \bar{x}+\bar{\epsilon}) \subset S_{3} .
$$

Since $\left[k_{1}, k_{2}\right]$ is compact, it follows from Lemma 4.8 and continuity of solutions with respect to initial conditions and parameters that there exist $\delta>0$ and $\bar{\epsilon}>0$ such that

$$
\delta<U^{\prime}(x, k)<\infty \quad \forall x \in[\bar{x}, \bar{x}+\bar{\epsilon}] \text { and } \forall k \in\left[k_{1}, k_{2}\right] .
$$

Thus, $U(x, k)$ exists for all $x \in[\bar{x}, \bar{x}+\bar{\epsilon})$ and for all $k \in\left[k_{1}, k_{2}\right]$, hence $[\bar{x}, \bar{x}+\bar{\epsilon}) \subset S_{3}$ as claimed.

Next, we show that $S_{3} \neq \phi$. Ignoring the negative terms in (4.85), we conclude that

$$
\left((p-1) U^{2}+1\right) U^{\prime} \leq-k p\left(U^{2}+1\right)^{2}+p\left(U^{2}+1\right) .
$$

Dividing both sides by $(p-1) U^{2}+1$, and using the fact that $-k p \leq-k_{1} p$, gives

$$
U^{\prime} \leq \frac{U^{2}+1}{(p-1) U^{2}+1}\left(-p k_{1}\left(U^{2}+1\right)+p\right) .
$$

Next, note that we have

$$
0<\frac{U^{2}+1}{(p-1) U^{2}+1} \leq \frac{p}{p-1} .
$$

Combining (4.116) and (4.117), we conclude that

$$
U^{\prime} \leq \frac{p^{2}}{p-1}\left(-k_{1}\left(U^{2}+1\right)+1\right) \leq \frac{p^{2}}{p-1}\left(-2 k_{1}+1\right)
$$


for $x \in\left[0, \frac{\pi}{2}\right)$ as long as $0 \leq U(x) \leq 1$. An integration gives

$$
U(x) \leq 1 \quad \forall x \in\left[0, \frac{p-1}{p^{2}\left(-2 k_{1}+1\right)}\right] \text { and } \forall k \in\left[k_{1}, k_{2}\right] .
$$

Because $p>1$, it is easily verified that $\frac{p-1}{p^{2}\left[-2 k_{1}+1\right)}<\frac{\pi}{2}$. This fact and (4.119) imply that

$$
\left(0, \frac{p-1}{p^{2}\left[-2 k_{1}+1\right)}\right] \subset S_{3} .
$$

This proves that $S_{3} \neq \phi$. It remains to show that $S_{3}=\left(0, \frac{\pi}{2}\right)$. Suppose, however, that

$$
x_{*}=\sup S_{3}<\frac{\pi}{2} \text {. }
$$

Then there exists $k_{*} \in\left(k_{1}, k_{2}\right)$ such that $U\left(x, k_{*}\right)$ ceases to exist at $x_{*}$; hence, it must be the case that $x_{k_{*}}=x_{*}$ and $U\left(x, k_{*}\right) \rightarrow \infty$ as $x \rightarrow x_{*}^{-}$. Since $U\left(x, k_{1}\right)$ is finite for all $x \in\left[0, x_{*}\right]$, we conclude that

$$
\lim _{x \rightarrow x_{*}^{-}}\left(U\left(x, k_{*}\right)-U\left(x, k_{1}\right)\right)=\infty .
$$

At $x=0$, it follows from (4.85) and (4.96) that

$$
U\left(0, k_{*}\right)-U\left(0, k_{1}\right)=0 \text { and } U^{\prime}\left(0, k_{*}\right)-U^{\prime}\left(0, k_{1}\right)=\left(k_{*}-k_{1}\right) \frac{(1-p)}{N-1}<0 .
$$

From (4.122) and (4.123) we conclude that there is an $\tilde{x} \in\left(0, x_{*}\right)$ such that

$$
U\left(x, k_{*}\right)-U\left(x, k_{1}\right)<0 \quad \forall x \in(0, \tilde{x}) \text { and } U\left(\tilde{x}, k_{*}\right)-U\left(\tilde{x}, k_{1}\right)=0 .
$$

However, since $\tilde{x}<x_{*}$, it follows from (4.110) and continuity that

$$
U\left(\tilde{x}, k_{*}\right)-U\left(\tilde{x}, k_{1}\right)=\int_{k_{1}}^{k_{*}} \frac{\partial U(\tilde{x}, k)}{\partial k} d k<0,
$$

contradicting (4.124). We conclude that

$$
S_{3}=\left(0, \frac{\pi}{2}\right)
$$

as claimed. Finally, it follows from inequality (4.105), the definition of $S_{3}$, (4.126) and continuity that

$$
\frac{\partial U(x, k)}{\partial k}<0 \quad \forall k \in\left[k_{1}, k_{2}\right] \text { and } \forall x \in\left(0, \frac{\pi}{2}\right) .
$$

\section{The Final Step (V)}

Case (a) Let $1<p<N$ and assume that there exist $k_{1}<k_{2}<\frac{N-p}{1-p}<0$, and corresponding solutions $y_{1}$ and $y_{2}$ of (4.6)-(4.7) which satisfy (4.8). It follows from (4.7), (4.8) and Lemma 4.7 that there exist values $\lambda_{1}>0$ and $\lambda_{2}>0$ such that

$$
\begin{aligned}
& y_{1}(0)=y_{2}(0)=1, y_{1}^{\prime}(0)=y_{2}^{\prime}(0)=0, \\
& y_{1}\left(\frac{\pi}{2}\right)=y_{2}\left(\frac{\pi}{2}\right)=0, y_{1}^{\prime}\left(\frac{\pi}{2}\right)=-\lambda_{1}<0 \text { and } y_{2}^{\prime}\left(\frac{\pi}{2}\right)=-\lambda_{2}<0 .
\end{aligned}
$$


Next, we conclude from (4.110) and continuity that

$$
U\left(x, k_{2}\right)-U\left(x, k_{1}\right)=\int_{k_{1}}^{k_{2}} \frac{\partial U(x, k)}{\partial k} d k<0 \quad \forall x \in\left(0, \frac{\pi}{2}\right) .
$$

Substituting $U\left(x, k_{1}\right)=\frac{1}{k_{1}} \frac{y_{1}^{\prime}(x)}{y_{1}(x)}$ and $U\left(x, k_{2}\right)=\frac{1}{k_{2}} \frac{y_{2}^{\prime}(x)}{y_{2}(x)}$ into (4.130), we obtain

$$
\frac{1}{k_{1}} \frac{y_{1}^{\prime}(x)}{y_{1}(x)}>\frac{1}{k_{2}} \frac{y_{2}^{\prime}(x)}{y_{2}(x)}>0 \quad \forall x \in\left(0, \frac{\pi}{2}\right) .
$$

An integration, together with (4.128), gives

$$
y_{1}(x)-\left(y_{2}(x)\right)^{\frac{k_{1}}{k_{2}}} \leq 0 \quad \forall x \in\left[0, \frac{\pi}{2}\right) .
$$

Since $\frac{k_{1}}{k_{2}}>1$, it follows from (4.129) that

$$
\left.\frac{\mathrm{d}}{\mathrm{d} x}\left(y_{1}(x)-\left(y_{2}(x)\right)^{\frac{k_{1}}{k_{2}}}\right)\right|_{x=\frac{\pi}{2}}=-\lambda_{1}<0 .
$$

We conclude from (4.129) and (4.133) there is an $\epsilon>0$ such that

$$
y_{1}(x)-\left(y_{2}(x)\right)^{\frac{k_{1}}{k_{2}}}>0 \text { for all } x \in\left(\frac{\pi}{2}-\epsilon, \frac{\pi}{2}\right),
$$

contradicting (4.132). Thus, $k_{1}$ and $k_{2}$ cannot exist and the proof is complete.

Case (b) For $p \geq N$, assume that there exist $k_{1}<k_{2}<0$, and corresponding solutions $y_{1}$ and $y_{2}$ of (4.6)-(4.7) which satisfy (4.8). A contradiction is obtained in exactly the same way as in Case (a), and we omit the details for brevity.

\section{References}

1. Aikawa, H., Kilpelinen, T., Shanmugalingam, N., Zhong, X.: Boundary Harnack principle for $p$-harmonic functions in smooth Euclidean domains. Potential Anal. 26(3), 281-301 (2007)

2. Aronsson, G.: Construction of singular solutions to the $p$-harmonic equation and its limit equation for $p=\infty$. Manuscr. Math. 56(2), 135-158 (1986)

3. Coddington, E.A., Levinson, N.: Theory of Ordinary Differential Equations. McGraw-Hill, New York (1955)

4. Coffman, C.: Uniqueness of the ground state solution for $\Delta u-u=u^{3}=0$ and a variational charcterization of other solutions. Arch. Ration. Mech. Anal. 46(2), 81-95 (1972)

5. Deblassie, D., Smits, R.G.: The p-harmonic measure of a small spherical cap. Le Mathematiche LXXIFasc I, 149-171 (2016)

6. DeBlassie, D., Smits, R.G.: The $p$-harmonic measure of small axially symmetric sets. Potential Anal. https://doi.org/10.1007/s11118-017-9668-0

7. Hartman, P.: Ordinary Differential Equations, Classics in Applied Mathematics. SIAM, Philadelphia (2002)

8. Hastings, S.P., McLeod, J.B.: Classical methods in ordinary differential equations: with applications to boundary value problems. In: Graduate Studies in Mathematics. Applied mathematics, vol. 129. American Mathematical Society (2011)

9. Heinonen, J., Kilpelinen, T., Martio, O.: Nonlinear Potential Theory of Degenerate Elliptic Equations. Dover, New York (1993)

10. Hirata, K.: Global estimates for non-symmetric Green type functions with application to the p-Laplace equation. Potential Anal. 29, 221-239 (2008)

11. Kwong, M.: Uniqueness of the positive solution of $\Delta u-u=u^{p}=0$ in $R_{n}$. Arch. Ration. Mech. Anal. 105, 3, 243-266 (1989)

12. Lewis, J., Nyström, K.: Boundary behaviour and the Martin boundary problem for $p$-harmonic functions in Lipschitz domains. Ann. Math. 172(3), 1917-1948 (2010) 
13. Lundstrom, N.L.P., Vasilis, J.: Decay of p-harmonic measure in the plane. Annales Academiae Scientiarum Fennicae Mathematica 38, 351-356 (2013)

14. Llorente, J.G., Manfredi, J.J., Wu, J.M.: p-Harmonic measure is not additive on null sets. Ann. Scuola Norm. Sup. Pisa Cl. Sci. IV, 357-373 (2005)

15. Martin, R.: Minimal positive harmonic functions. Trans. Am. Math. Soc. 49, 137-172 (1941)

16. McLeod, K., Troy, W.C., Weissler, F.: Radial solutions of $\Delta u+f(u)=0$ with prescribed numbers of zeros. J. Differ. Eqs. 83, 368-378 (1990)

17. Nehari, Z.: On a nonlinear differential equation arising in nuclear physics. Proc. R. Ir. Acad. 62, 117-135 (1963)

18. Peres, Y., Schramm, O., Sheffield, S., Wilson, D.B.: Tug-of-war and the infinity Laplacian. J. Am. Math. Soc. 22, 167-210 (2009)

19. Porretta, A., Véron, L.: Separable $p$-harmonic functions in a cone and related quasilinear equations on manifolds. J. Eur. Math. Soc. 11, 1285-1305 (2009)

20. Tolksdorf, P.: On the Dirichlet problem for quasilinear equations in domains with conical boundary points. Comm. Partial Differ. Equ. 8, 773-817 (1983)

21. Zhang, Z., Lie, S.: On sign-changing and multiple solutions of the p-Laplacian. J. Funct. Anal. 197, 447-468 (2003)

Publisher's Note Springer Nature remains neutral with regard to jurisdictional claims in published maps and institutional affiliations. 